\title{
ChEC-seq produces robust and specific maps of transcriptional regulators
}

\section{Gabriel E. Zentner ${ }^{1,2}$, Robert A. Policastro', Steven Henikoff ${ }^{3,4}$}

${ }^{1}$ Department of Biology, Indiana University, Bloomington, IN 47405, USA

${ }^{2}$ Indiana University Melvin and Bren Simon Cancer Center, Indianapolis, IN 46202

${ }^{3}$ Basic Sciences Division, Fred Hutchinson Cancer Research Center, Seattle, WA 98109, USA

${ }^{4}$ Howard Hughes Medical Institute, Fred Hutchinson Cancer Research Center, Seattle, WA 98109, USA

Correspondence to G. E. Z. (gzentner@indiana.edu) or S.H. (steveh@fredhutch.org) 


\begin{abstract}
We previously introduced chromatin endogenous cleavage and high-throughput sequencing (ChEC-seq), which uses fusion of a chromatin-binding protein of interest to micrococcal nuclease (MNase) and high-throughput sequencing to generate genomewide maps of factor binding. Here, we respond to concerns that have been raised about the specificity of the method relative to its negative control when a single long calcium incubation time is used. Through reanalysis of our previously published datasets, we show that short-duration ChEC-seq experiments provide robust, specific maps of transcriptional regulators across the budding yeast genome. Our analyses also confirm that consideration of MNase digestion kinetics is important for proper design and interpretation of ChEC-seq experiments.
\end{abstract}


Pugh and colleagues report little difference between signals for MNase-tagged proteins and free MNase (that is, nuclear-localized MNase not fused to any factor) when a single long time point (5 minutes after calcium addition) is analyzed (Mittal et al., 2021). Enrichment is thus considered to be largely nonspecific. We originally performed a broad time course analysis of cleavage by the MNase-tagged general regulatory factors (GRFs) Abf1, Rap1, and Reb1 and free MNase. This revealed two general classes of binding sites: 'fast' sites, where maximal cleavage was reached $\leq 1$ minute after calcium addition, and 'slow' sites, where cleavage increased up to 20 minutes after calcium addition. Thus, consideration of MNase cleavage kinetics is important for proper interpretation of ChEC-seq results.

Expression of free MNase in cells followed by calcium incubation is akin to the use of exogenous MNase in a standard epigenome profiling experiment, with diffusion, chromatin association, and cleavage kinetics unconstrained by fusion to a protein with affinity for specific loci. Thus, longer digestion times yield increased cleavage within accessible DNA, resulting in increased 'nonspecific' signal that negates the remaining specific signal. To illustrate this, we plotted free MNase signal around the TSSs of 4,857 genes encoding verified ORFs across a 40 -fold range of calcium incubation times. We observed weak signal at 30 seconds and 1 minute of incubation with calcium (Fig. 1A-B), but by 5 minutes noted robust cleavage within promoter NDRs and nucleosome linkers, leading to an uneven background.

Having established that short calcium incubations produce relatively uniform background cleavages by free MNase, we assessed the binding of GRFs and Mediator at genes classified as dominated by the SAGA or TFIID coactivator complexes in their regulation (Huisinga and Pugh, 2004) using merged $\leq 1$ minute calcium incubation time points for our previously published factor-specific and free MNase ChEC-seq datasets (Grünberg et al., 2016; Zentner et al., 2015). At both gene sets, we detected robust enrichment of all factors over free MNase on average (Fig. 1C).

We initially reported numbers of fast GRF binding sites lower than the approximate number of RNA polymerase II (RNAPII)-transcribed genes in the yeast genome (Abf1, 3,702; Rap1, 1,974; Reb1, 2,711) (Zentner et al., 2015) and did not annotate the genes associated with these sites. Thus, it was unclear how many genes are associated with rapid, specific cleavage by GRF-MNase fusions. To assess this, we have visualized GRF ChEC-seq and free MNase signal around TSSs as heatmaps. Approximately one-third of the analyzed TSSs displayed upstream enrichment of Abf1 
by ChEC-seq and CUT\&RUN (Skene and Henikoff, 2017) (Fig. 1D). Enrichment of Rap1 was observed at $\sim 15 \%$ of the analyzed TSSs by both ChEC-seq and ChIP-exo (Rossi et al., 2018) (Fig. 1E). We found robust Reb1 enrichment at $\sim 25 \%$ of analyzed TSSs by ChEC-seq and CUT\&RUN (Fig. 1F). We similarly assessed Mediator enrichment around TSSs. Approximately $25 \%$ of TSSs displayed strong upstream enrichment of both Med8 and Med17; however, modest signal was observed for most of the remaining TSSs relative to free MNase (Fig. $1 \mathrm{G}$ ). This pattern was also seen in no-tag-normalized Mediator ChIP-chip data (Jeronimo et al., 2016). We conclude that ChEC-seq indeed reveals widespread binding of Mediator to the upstream regions of most RNAPIItranscribed genes, consistent with the global negative effect of Mediator dissociation on RNAPII occupancy (Warfield et al., 2017). Visualization of all datasets at the loci examined in Fig. 1D-G also revealed common and distinct peaks (Fig. 2), supporting capture of specific binding events by short-duration ChEC-seq experiments.

We next called peaks of GRF ChEC-seq binding and assessed motif enrichment. We observed bimodal enrichment of known motifs around peak summits, consistent with cleavage of DNA adjacent to the motif, and detected robust consensus sequences in peaks de novo (Fig. 3A-C). Short-duration ChEC-seq experiments thus capture sequence-specific binding of GRFs, consistent with our detection of strong motifs in 'fast' sites (Zentner et al., 2015). We also determined the fraction of reads in peaks (FRiP) (Landt et al., 2012) for each dataset after down-sampling and peak calling. All three GRF ChEC-seq datasets displayed exceptional signal-to-noise ratios, with FRiP values of 0.45-0.65 obtained using just $\sim 25,000$ reads for peak calling (Fig. 3D). Numbers of called peaks increased with additional reads considered and plateaued at $\sim 2$ million reads with $\mathrm{FRiP}$ values of $0.69-0.84$, indicating that at least two-thirds of all reads from each dataset is contained within peaks called using $\sim 2$ million reads. Together with the above analyses of motif enrichment, these results confirm that short-duration ChEC-seq produces high-quality data for GRFs.

We have shown that short-duration calcium incubations yield robust and specific signal for GRFs and Mediator as measured by enrichment over free MNase, signal from orthogonal methods, and the presence of consensus motifs (for GRFs). The apparent lack of specificity in ChEC-seq experiments in which long calcium incubation is used is likely due to two additive factors - decreased specific signal in the ChEC-seq experiment and increased nonspecific signal in the free MNase experiment. Detection of these factors was made possible by the use of time courses for both factor-specific and free 
MNase experiments, as performed in the original ChEC-seq paper (Zentner et al., 2015) and recommended in a related methods article (Grünberg and Zentner, 2017). Given the increase in free MNase signal at longer time points, comparison of short-duration MNase fusion and free MNase experiments ( $\leq 1$ minute) yields the most robust results. Similar observations were made in a recent study that used ChEC-seq to characterize the contribution of intrinsically disordered regions of transcription factors to their DNAbinding activities (Brodsky et al., 2020). The authors performed a calcium digestion time series and chose short incubation times $(30-60 \mathrm{sec})$ for their experiments.

As a complement to this article, we provide a detailed description of our current best practices for ChEC-seq at protocols.io (https://www.protocols.io/view/chromatinendogenous-cleavage-and-high-throughput-bgthjwj6). We also draw attention to two additional responses to these critiques from the Hahn (Donczew et al, bioRxiv 2021) and Shore (Bruzzone et al, bioRxiv 2021) labs.

\section{Methods}

\section{Datasets}

FASTQ files were obtained from the SRA: GRF and W1588-4C free MNase ChEC-seq (SRP056746) (Zentner et al., 2015); Mediator and BY4705 free MNase ChEC-seq (SRP074777) (Grünberg et al., 2016); Abf1 and Reb1 CUT\&RUN (SRP078609) (Skene and Henikoff, 2017); Rap1 ChIP-exo (SRP096827) (Rossi et al., 2018). The MetaMediator track was obtained from the supplemental of the original publication (Jeronimo et al., 2016) and converted to a bigWig file with UCSC bedGraphToBigWig.

\section{Read alignment and processing}

Paired FASTQ files were aligned to the sacCer3 genome assembly with Bowtie2 (Langmead and Salzberg, 2012) using default parameters plus '--no-unal --no-mixed -no-discordant --dovetail -I 10 -X 700'. Alignment SAM files were converted to sorted and indexed BAM files with SAMtools ( $\mathrm{Li}$ et al., 2009). BAM files were merged with SAMtools. For GRF ChEC-seq analysis, $10 \mathrm{~s}, 20 \mathrm{~s}, 30 \mathrm{~s}, 40 \mathrm{~s}, 50 \mathrm{~s}$, and $1 \mathrm{~m}$ time points were merged. For Mediator ChEC-seq analysis, $30 \mathrm{~s}$ and $1 \mathrm{~m}$ time points were merged. For CUT\&RUN analysis, 1 s, 2 s, 4 s, 8 s, 16 s, 32 s, 64 s, and 128 s time points were merged. For ChIP-exo analysis, four replicates of Rap1 ChIP-exo were merged. Unless otherwise specified, all downstream analyses were performed with components of deepTools2 (Ramírez et al., 2016). 


\section{Read coverage}

Read coverage bigWig files were generated with bamCoverage with a bin size of 1 and CPM normalization. For Figure $1 \mathrm{~A}-\mathrm{B}$ heatmaps, 25 bp reads were not extended, allowing sharper visualization of periodic gene body signal. Reads were otherwise extended using bamCoverage -e, which extends reads to their fragment length based on mate-pair distance. CUT\&RUN coverage was computed using the additional flag '-maxFragmentLength 120' to exclude nucleosomal fragments as described in the original CUT\&RUN publication (Skene and Henikoff, 2017). Genome browser visualization was performed with Gviz (Hahne and Ivanek, 2016).

\section{Heatmaps and average plots}

The list of 4,857 TSSs used was generated by intersecting a published TSS list (Xu et al., 2009) with the verified ORF list from the Saccharomyces Genome Database. This list was then intersected with a list of SAGA/TFIID classifications (Huisinga and Pugh, 2004), yielding TSSs of 436 SAGA-dominated and 4,052 TFIID-dominated genes. TSS lists were then used to generate matrices of signal with computeMatrix. Heatmaps were generated with plotHeatmap. Average plot values were obtained with plotProfile and plotted in GraphPad Prism 7.

\section{Peak calling}

GRF peaks were called using MACS2 (Zhang et al., 2008) with the merged W1588-4C free MNase dataset as the control file and an effective genome size of $12,100,000$. To calculate FRiP values, GRF ChEC-seq alignment BAM files were downsampled with SAMtools and peaks were called as above. Fragments in peaks were counted with the featureCounts tool of Rsubread (Liao et al., 2019) and the assignment status of each fragment was output in CORE format. A fragment was required to have at least $50 \mathrm{bp}$ overlap with a peak to be assigned to that peak. The fraction of fragments assigned to a peak was considered to be the FRiP for that dataset. BAM downsampling, peak calling, and read assignment to peaks were automated using a custom conda environment with bash and R scripts (https://github.com/rpolicastro/chip downsampling).

\section{Motif analysis}


To determine motif enrichment around peak summits, we used HOMER (http://homer.ucsd.edu) (Heinz et al., 2010) annotatePeaks.pl in motif finding mode ('-m') with $10 \mathrm{bp}$ bins and the corresponding recommended position frequency matrix from ScerTF (Spivak and Stormo, 2012) after conversion to HOMER format. Position frequency matrices were derived from the following publications: Abf1 (Maclsaac et al., 2006), Rap1 (Morozov and Siggia, 2007), Reb1 (Badis et al., 2008). De novo motif searching was performed with HOMER findMotifsGenome.pl with a $400 \mathrm{bp}$ window around peak summits with 6,516 yeast promoters $(-1000$ to +100 bp relative to the TSS with chrM sequences removed) as background regions. Based on the widths of recommended consensus motifs from ScerTF, motif size ranges of 13-17 bp, 11-15 bp, and 7-11 bp for Abf1, Rap1, and Reb1, respectively, were used.

\section{Acknowledgements}

We thank Jorja Henikoff for analytical assistance. This work was supported by $\mathrm{NIH}$ grants R35GM128631 (G.E.Z.) and R01HG010492 (S.H.) and the Howard Hughes Medical Institute (S.H.) 


\section{References}

Badis, G., Chan, E.T., van Bakel, H., Pena-Castillo, L., Tillo, D., Tsui, K., Carlson, C.D., Gossett, A.J., Hasinoff, M.J., Warren, C.L., et al. (2008). A Library of Yeast Transcription Factor Motifs Reveals a Widespread Function for Rsc3 in Targeting Nucleosome Exclusion at Promoters. Mol. Cell 32, 878-887.

Brodsky, S., Jana, T., Mittelman, K., Chapal, M., Kumar, D.K., Carmi, M., and Barkai, N. (2020). Intrinsically Disordered Regions Direct Transcription Factor In Vivo Binding Specificity. Molecular Cell 79, 459-471.e454.

Grünberg, S., Henikoff, S., Hahn, S., and Zentner, G.E. (2016). Mediator binding to UASs is broadly uncoupled from transcription and cooperative with TFIID recruitment to promoters. The EMBO Journal 35, 2435-2446.

Grünberg, S., and Zentner, G.E. (2017). Genome-wide Mapping of Protein-DNA Interactions with ChEC-seq in Saccharomyces cerevisiae. Journal of Visualized Experiments, e55836.

Hahne, F., and Ivanek, R. (2016). Visualizing Genomic Data Using Gviz and Bioconductor. In Statistical Genomics: Methods and Protocols, E. Mathé, and S. Davis, eds. (New York, NY: Springer New York), pp. 335-351.

Heinz, S., Benner, C., Spann, N., Bertolino, E., Lin, Y.C., Laslo, P., Cheng, J.X., Murre, C., Singh, H., and Glass, C.K. (2010). Simple Combinations of Lineage-Determining Transcription Factors Prime cis-Regulatory Elements Required for Macrophage and $B$ Cell Identities. Mol. Cell 38, 576-589.

Huisinga, K.L., and Pugh, B.F. (2004). A Genome-Wide Housekeeping Role for TFIID and a Highly Regulated Stress-Related Role for SAGA in Saccharomyces cerevisiae. Mol. Cell 13, 573-585.

Jeronimo, C., Langelier, M.-F., Bataille, Alain R., Pascal, John M., Pugh, B.F., and Robert, F. (2016). Tail and Kinase Modules Differently Regulate Core Mediator Recruitment and Function In Vivo. Mol. Cell 64, 455-466.

Landt, S.G., Marinov, G.K., Kundaje, A., Kheradpour, P., Pauli, F., Batzoglou, S., Bernstein, B.E., Bickel, P., Brown, J.B., Cayting, P., et al. (2012). ChIP-seq guidelines 
and practices of the ENCODE and modENCODE consortia. Genome Res. 22, 18131831.

Langmead, B., and Salzberg, S.L. (2012). Fast gapped-read alignment with Bowtie 2. Nat. Meth. 9, 357-359.

Li, H., Handsaker, B., Wysoker, A., Fennell, T., Ruan, J., Homer, N., Marth, G., Abecasis, G., Durbin, R., and Subgroup, G.P.D.P. (2009). The Sequence Alignment/Map format and SAMtools. Bioinformatics 25, 2078-2079.

Liao, Y., Smyth, G.K., and Shi, W. (2019). The R package Rsubread is easier, faster, cheaper and better for alignment and quantification of RNA sequencing reads. Nucleic Acids Res. 47, e47-e47.

Maclsaac, K., Wang, T., Gordon, D.B., Gifford, D., Stormo, G., and Fraenkel, E. (2006). An improved map of conserved regulatory sites for Saccharomyces cerevisiae. BMC Bioinformatics 7, 113.

Mittal, C., Rossi, M.J., and Pugh, B.F. (2021). High similarity among ChEC-seq datasets. bioRxiv https://doi.org/10.1101/2021.02.04.429774.

Morozov, A.V., and Siggia, E.D. (2007). Connecting protein structure with predictions of regulatory sites. Proceedings of the National Academy of Sciences 104, 7068-7073.

Ramírez, F., Ryan, D.P., Grüning, B., Bhardwaj, V., Kilpert, F., Richter, A.S., Heyne, S., Dündar, F., and Manke, T. (2016). deepTools2: a next generation web server for deepsequencing data analysis. Nucleic Acids Res. 44, W160-W165.

Rossi, M.J., Lai, W.K.M., and Pugh, B.F. (2018). Genome-wide determinants of sequence-specific DNA binding of general regulatory factors. Genome Res. 28, 497-508.

Skene, P.J., and Henikoff, S. (2017). An efficient targeted nuclease strategy for highresolution mapping of DNA binding sites. eLife 6 , e21856.

Spivak, A.T., and Stormo, G.D. (2012). ScerTF: a comprehensive database of benchmarked position weight matrices for Saccharomyces species. Nucleic Acids Res. 40, D162-D168. 
Warfield, L., Ramachandran, S., Baptista, T., Devys, D., Tora, L., and Hahn, S. (2017). Transcription of Nearly All Yeast RNA Polymerase II-Transcribed Genes Is Dependent on Transcription Factor TFIID. Mol. Cell 68, 118-129.

Xu, Z., Wei, W., Gagneur, J., Perocchi, F., Clauder-Munster, S., Camblong, J., Guffanti, E., Stutz, F., Huber, W., and Steinmetz, L.M. (2009). Bidirectional promoters generate pervasive transcription in yeast. Nature 457, 1033-1037.

Zentner, G.E., Kasinathan, S., Xin, B., Rohs, R., and Henikoff, S. (2015). ChEC-seq kinetics discriminates transcription factor binding sites by DNA sequence and shape in vivo. Nat Commun 6, 8733.

Zhang, Y., Liu, T., Meyer, C.A., Eeckhoute, J., Johnson, D.S., Bernstein, B.E., Nusbaum, C., Myers, R.M., Brown, M., Li, W., et al. (2008). Model-based Analysis of ChIP-Seq (MACS). Genome Biol. 9, R137. 
A

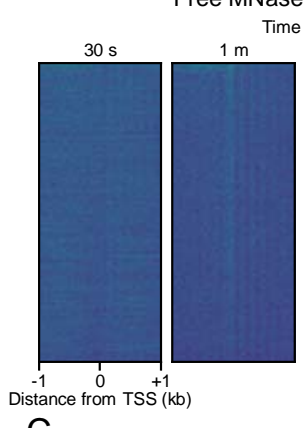

C
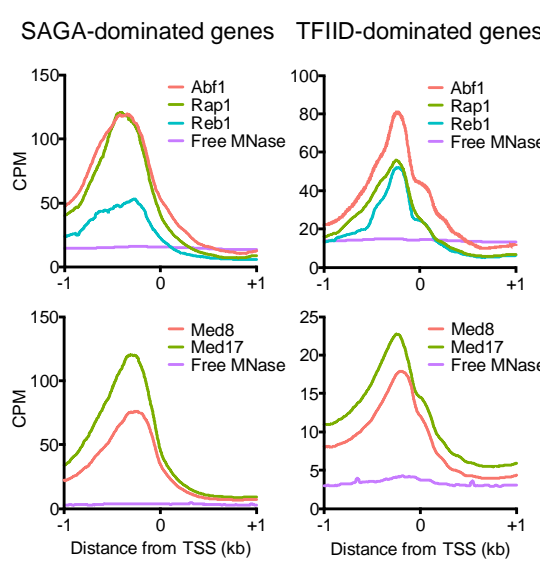

$\mathrm{F}$

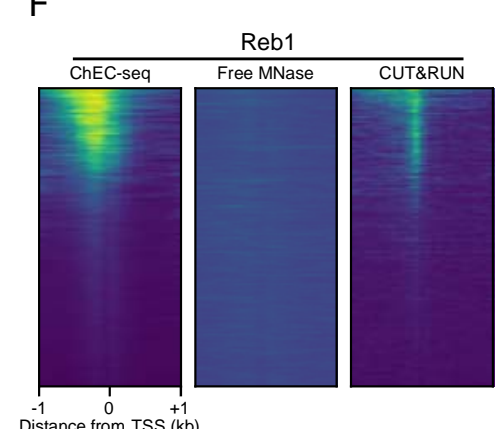

Distance from TSS $\stackrel{+1}{(k b)}$
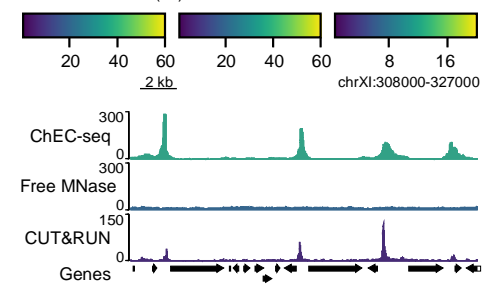

B Free MNase (MED8 promoter-driven)

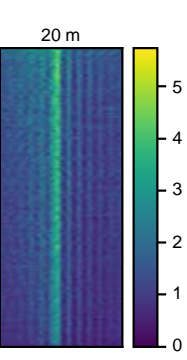

$\mathrm{D}$

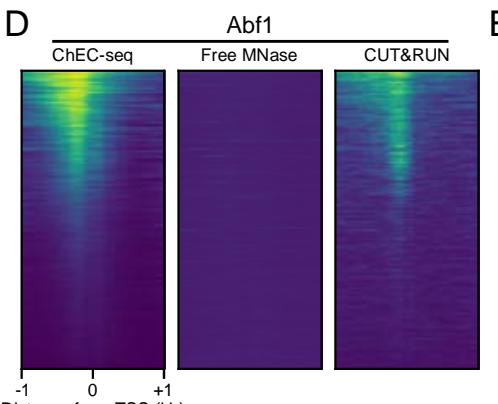

$\mathrm{E}$
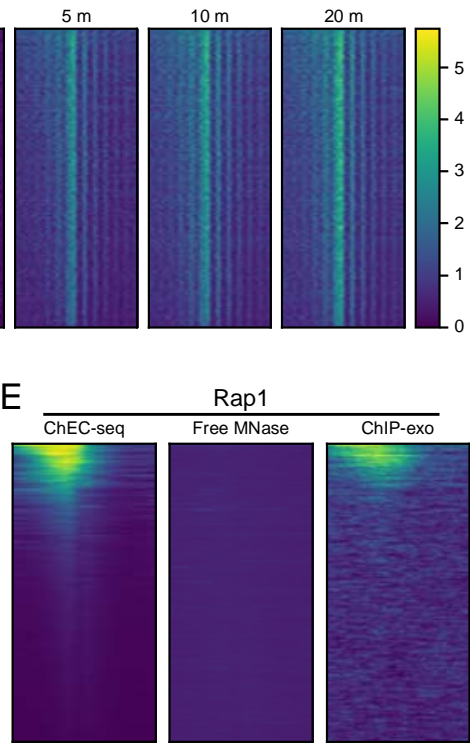

Distance from $\operatorname{TSS}_{(\mathrm{kb})}^{+1}$
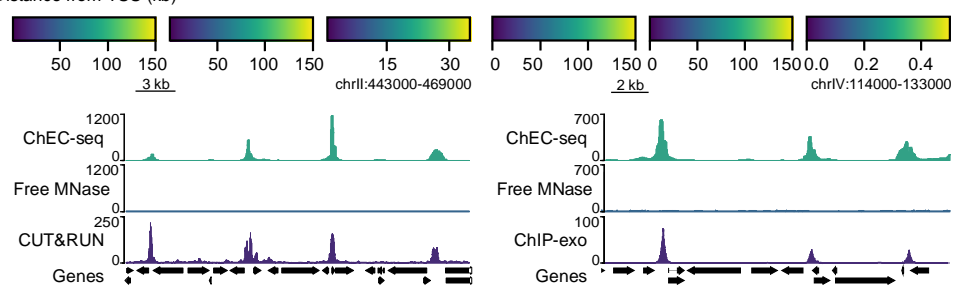

G
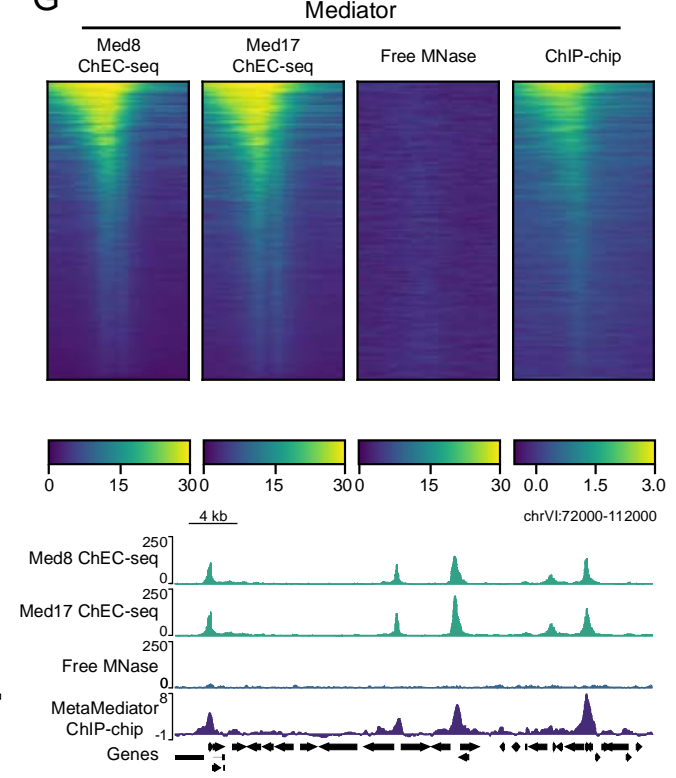

\section{Figure 1. Short-duration ChEC-seq experiments show high specificity}

Heatmaps of CPM-normalized free MNase signal around 4,857 TSSs. Each heatmap is independently sorted descending by the average signal in the $2 \mathrm{~kb}$ window surrounding each TSS. (C) Average plots of CPM-normalized GRF (merged $10 \mathrm{~s}-1 \mathrm{~m}$ time points), 
Mediator subunit (merged $30 \mathrm{~s}$ and $1 \mathrm{~m}$ time points), and free MNase (merged $10 \mathrm{~s}-1$ $\mathrm{m}$ time points for W1588-4C, merged $30 \mathrm{~s}$ and $1 \mathrm{~m}$ time points for BY4705) signal at SAGA-dominated and TFIID-dominated genes. (D) Heatmaps of pooled $10 \mathrm{~s}-1 \mathrm{~m}$ Abf1 and W1588-4C free MNase ChEC-seq and pooled $1 s-128 s$ Abf1 CUT\&RUN at 4,857 TSSs. All heatmaps are sorted descending by average Abf1 ChEC-seq signal in the $2 \mathrm{~kb}$ window surrounding each TSS. Also shown are genome browser tracks of the same data along a representative segment of the yeast genome. (E) Same as (D) but for Rap1 and with pooled Rap1 ChIP-exo signal. (F) Same as (D) but for Reb1. (G) Heatmaps of pooled $30 \mathrm{~s}$ and $1 \mathrm{~m}$ Med8, Med17, and BY4705 free MNase ChEC-seq and MetaMediator (combined ChIP-chip signal for 12 Mediator subunits) data from WT yeast. All heatmaps are sorted descending by average Med8 ChEC-seq signal in the $2 \mathrm{~kb}$ window surrounding each TSS. Also shown are genome browser-style tracks of the same data along a representative segment of the yeast genome. See Fig. 2 for genome browser-style views of all analyzed datasets at each region shown here. 

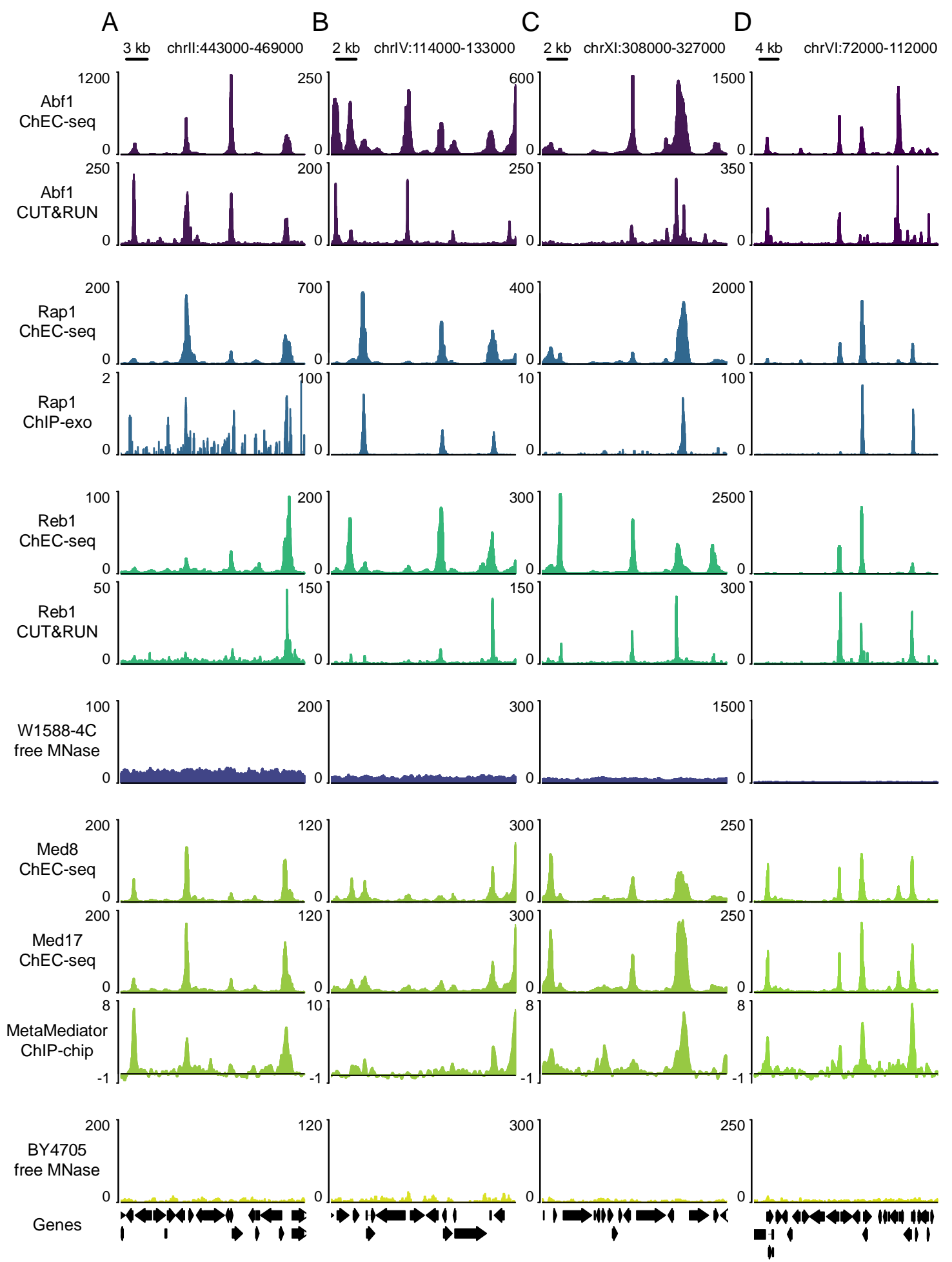

Figure 2. Landscapes of GRF and Mediator binding to the yeast genome

Genome browser-style visualization of all analyzed ChEC-seq, CUT\&RUN, and ChIPexo datasets at the four regions of the yeast genome displayed in the analysis of (A) Abf1, (B) Rap1, (C) Reb1, and (D) Mediator ChEC-seq data presented in Fig. 1D-G. 
A

Abf1

$\square$ Motif density

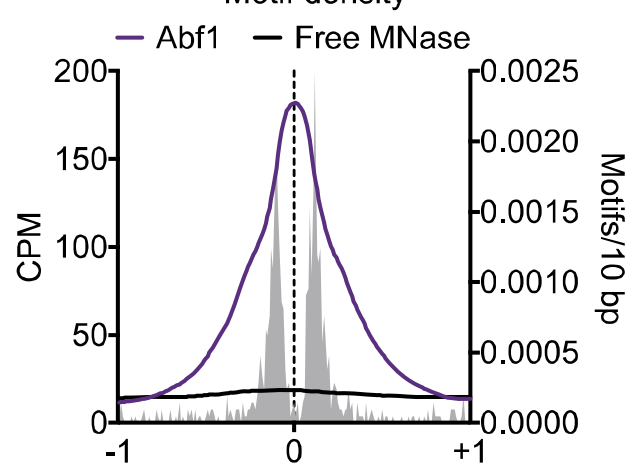

Distance from peak summit $(\mathrm{kb})$

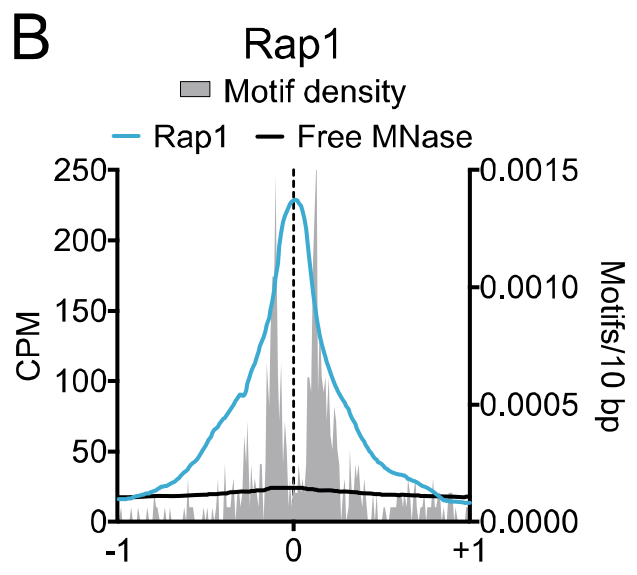

Distance from peak summit $(k b)$

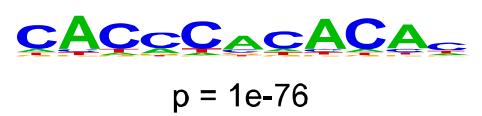

$\mathrm{D}$

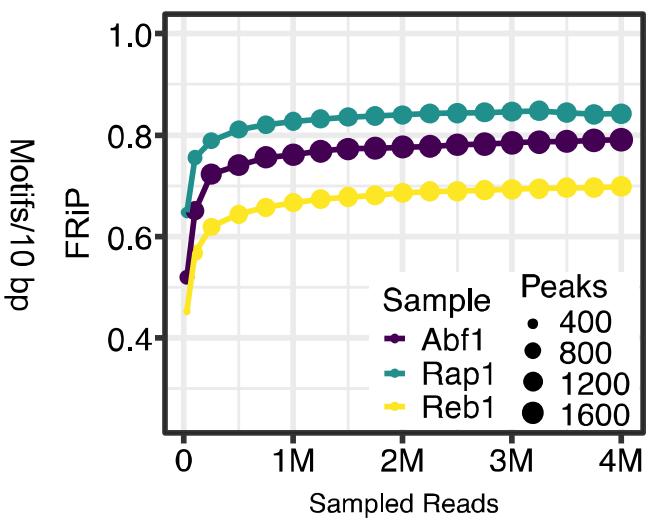

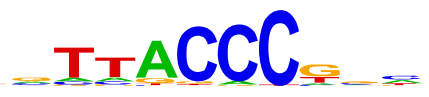

$p=1 e-883$

Figure 3. ChEC-seq detects sequence-specific binding of GRFs

Average plots showing ChEC-seq signal and motif enrichment in $10 \mathrm{bp}$ bins around the summits of (A) 2,098 Abf1 peaks, (B) 1,623 Rap1 peaks, and (C) 1,564 Reb1 peaks. The sequence logo of the top motif match discovered de novo with peaks for the indicated factor is shown below each plot. (D) Fraction of reads in peaks (FRiP) values and peak numbers for down-sampled GRF ChEC-seq datasets. Down-sampled read numbers were $25,000,100,000$, and then every 250,000 starting at 250,000. 